\title{
Ellipticity and deviations from orthogonality in the polarization modes of PSR B0329+54^
}

\begin{abstract}
R. T. Edwards ${ }^{1}$ and B. W. Stappers ${ }^{1,2}$
1 Astronomical Institute "Anton Pannekoek”, University of Amsterdam, Kruislaan 403, 1098 SJ Amsterdam, The Netherlands

2 Stichting ASTRON, Postbus 2, 7990 AA Dwingeloo, The Netherlands

Received 9 February 2004 / Accepted 26 March 2004

Abstract. We report on an analysis of the polarization of single pulses of PSR B0329+54 at $328 \mathrm{MHz}$. We find that the distribution of polarization orientations in the central component diverges strongly from the standard picture of orthogonal polarization modes (OPMs), making a remarkable partial annulus on the Poincare sphere. A second, tightly clustered region of density appears in the opposite hemisphere, at a point antipodal to the centre of the annulus. We argue that this can be understood in terms of birefringent alterations in the relative phase of two elliptically polarized propagation modes in the pulsar magnetosphere (i.e. generalised Faraday rotation). The ellipticity of the modes implies a significant charge density in the plasma, while the presence of both senses of circular polarization, and the fact that only one mode shows the effect, supports the view that refracted ordinary-mode rays are involved in the production of the annulus. At other pulse longitudes the polarization (including the circular component) is broadly consistent with an origin in elliptical OPMs, shown here quantitatively for the first time, however considerable non-orthogonal contributions serve to broaden the orientation distribution in an isotropic manner.
\end{abstract}

Key words. plasmas - polarization - stars: pulsars: individual: PSR B0329+54 - waves

\section{Introduction}

Whenever sufficient sensitivity is available, radio pulsar emissions are seen to be rich in phenomenology. Their polarization is no exception to this rule. The dependence of linear polarization position angle on pulse longitude (i.e. rotational phase) can, for some pulsars, be explained as arising in the vicinity of the magnetic pole, polarized linearly at the position angle of the sky projection of magnetic field lines (Radhakrishnan \& Cooke 1969). For other pulsars this is not the case, and for some of these the distribution of position angles (PAs) in individual pulses has been shown to be bimodal about two values separated by $90^{\circ}$ - so-called orthogonal polarization modes (OPMs; e.g. Manchester et al. 1975; Backer et al. 1976; Cordes et al. 1978; Backer \& Rankin 1980; Stinebring et al. 1984a,b). Many pulsars also show $90^{\circ}$ "jumps" in their position angle profiles, a fact that received explanation in the discovery of OPMs through a longitude dependence of the relative intensities of the modes, which themselves tend to have position angle swings consistent with the magnetic pole model of Radhakrishnan \& Cooke (1969). These studies of OPMs also found evidence for deviations from orthogonality in the fact that PA distributions were broader than expected and/or their peaks were not separated by $90^{\circ}$.

Send offprint requests to: $\mathrm{R}$. T. Edwards,

e-mail: redwards@science.uva.nl

* Appendix A is only available at:

http://www.edpsciences.org
Attempts have been made to explain these deviations from orthogonality by means of superposition of two modes that are not orthogonal due to origins on different field lines and subsequent birefringent refraction (e.g. Stinebring et al. 1984a; McKinnon 2003a), by means of the superposition of a range of modal orientations arising from a distribution of field lines that are visible due to their finite beam width (Gil \& Lyne 1995), or due to the presence of two instantaneously orthogonal modes, the orientation of which varies with time due to coherent wave coupling effects (e.g. Cheng \& Ruderman 1979; Lyubarskii \& Petrova 1999). Of these, only the first and third scenarios have been quantitatively tested. McKinnon (2003a) showed that the distribution of PAs, and the shape of the average PA curve of PSR B2016+28 are consistent with the superposition of two non-orthogonal modes. On the other hand, Petrova (2003) showed that the PA and circular polarization of the average pulse profiles of PSR B0355+54 and PSR B0628-28 are consistent with the predictions of Lyubarskii \& Petrova (1999) for alterations in the polarization due to coherent effects. However, neither work examines whether the data are also consistent with other models, and indeed the statistics considered in each case are not well suited to distinguishing between models.

The question of the origin of circular polarization in pulsars is also not addressed by the magnetic pole model, in which the polarization is expected to be linear due to the high magnetic field strength. The fact that orthogonal linear polarization states have been found to be associated with opposite signs of circular polarization leads naturally to the suggestion that 
the modes are in fact elliptically polarized orthogonal modes (Cordes et al. 1978), although to our knowledge, to date no observational tests of the expected proportionality between linear and circular polarization under this hypothesis have been made. In explaining the origin of elliptical OPMs, most authors point to the so-called polarization-limiting region (PLR), where birefringent propagation effects no longer significantly alter the relative phase of the modes, as the determinant of the observed polarization (Cheng \& Ruderman 1979). It has been proposed that either the propagation modes themselves are elliptical at this point, requiring a net charge density in the plasma (Cheng \& Ruderman 1979; Allen \& Melrose 1982; von Hoensbroech et al. 1998), or that weak birefringence in the vicinity of the PLR causes initially linearly polarized rays to suffer changes in their polarization if their position angle deviates from that of the local linear modes, for example due to rotational aberration or refraction (Cheng \& Ruderman 1979; Lyubarskii \& Petrova 1999; Petrova \& Lyubarskii 2000; Petrova 2001, 2003).

In this work we make a detailed study of the polarization of single pulses from PSR B0329+54, employing new techniques in the hope of placing greater constraints on the nature and origin of ellipticity and non-orthogonality of pulsar polarization.

\section{Methods of analysis}

\subsection{Stokes parameters of OPM}

Since the focus of this study is the inconsistency of observations with a simple model of superposed OPMs, it is pertinent to begin with a clear picture of the features expected under such a model, before discussing ways in which the observations may deviate from it. The distribution of Stokes parameters expected under the incoherent superposition of two elliptical OPMs, and techniques for reconstruction of the modal intensities have been considered in detail by McKinnon \& Stinebring (2000).We briefly re-iterate in a more compact vector form before considering the fluctuation statistics.

The modes are taken to be completely polarized, with fixed position angles and degrees of circular polarization at a given pulse longitude. This means that the vector $\boldsymbol{p}_{i}=(Q U V)^{T}$ for a given mode $i$ always has the same orientation, regardless of its length (i.e. the intensity of the mode). The condition of orthogonality of the electric field vectors requires that the polarization states be antiparallel in the Poincaré sphere ( $\boldsymbol{p}$-space). Since the Stokes 4-vector of the incoherent sum of two polarization states is simply the sum of their respective Stokes vectors, all resultant states must lie on the line defined by the modal orientation. The observed Stokes parameters at any instant can be written as:

$$
\begin{aligned}
I & =I_{1}+I_{2} \\
\boldsymbol{p} & =p_{1}+p_{2} \\
& =\left(I_{1}-I_{2}\right) \mathbf{1}_{\boldsymbol{m}},
\end{aligned}
$$

where the contributions from the two modes are denoted with numerical subscripts, with $\mathbf{1}_{\boldsymbol{m}}$ being a unit vector in the direction associated with the mode denoted " 1 ".
The procedure of "mode separation" (determination of modal intensities) follows directly as:

$I_{1}=(I+|p|) / 2$

$I_{2}=(I-|p|) / 2$,

while the modal orientation is simply given by $\mathbf{1}_{\boldsymbol{m}}=\boldsymbol{p} /|\boldsymbol{p}|$. Here we have explicitly identified mode 1 as the greater in intensity. Note that $|\boldsymbol{p}|$ is subject to bias in the presence of measurement noise. Although this can be corrected, if some alternative means is available for determining the modal orientation, since components of $\boldsymbol{p}$ orthogonal to $\mathbf{1}_{\boldsymbol{m}}$ contribute only noise to $|\boldsymbol{p}|$ one may eliminate the bias and improve sensitivity by substituting $\boldsymbol{p} \cdot \mathbf{1}_{\boldsymbol{m}}$ for $|\boldsymbol{p}|$. Prior information on $\mathbf{1}_{\boldsymbol{m}}$ could, for example, be obtained from studies of the statistics of $\boldsymbol{p}$ (Sect. 2.3.1), or for mode-separation of individual pulses, from the average profile.

By measuring appropriate statistics of the Stokes vectors, information is available on the fluctuation statistics of the OPMs. Characterising the latter by the modal variances $\sigma_{1}^{2}$ and $\sigma_{2}^{2}$ and their covariance $\sigma_{12}$, one observes that

$\sigma_{I}^{2}=\sigma_{1}^{2}+\sigma_{2}^{2}+2 \sigma_{12}$

$\sigma_{m}^{2}=\sigma_{1}^{2}+\sigma_{2}^{2}-2 \sigma_{12}$,

where $\sigma_{m}^{2}$ is the variance of $\boldsymbol{p} \cdot \mathbf{1}_{\boldsymbol{m}}$. From this the covariance of the modes can be calculated as

$\sigma_{12}=\left(\sigma_{I}^{2}-\sigma_{m}^{2}\right) / 4$

In practice the measured variances will be biased by the presence of measurement noise, which can be estimated using the off-pulse covariances (and the pulsar intensity, if it contributes appreciably to the system temperature), and subtracted.

\subsection{Characterising the distribution on the Poincaré sphere}

The defining feature of elliptical OPMs is the predicted constant orientation of $\boldsymbol{p}$, regardless of pulse-to-pulse fluctuations in $I$ and $|p|$. This fact is put to good use in the display of histograms of the position angle of linear polarization, $\psi=$ $\frac{1}{2} \tan ^{-1} U / Q$, which at a given longitude can take one of two allowed values offset by $90^{\circ}$. In the presence of instrumental noise these broaden to give the characteristic bimodal distribution of OPM. The position angle distribution gives information on the orthogonality of the linear component of the polarization, but the question must also be asked whether the circular component is as expected under elliptical OPM. Attempts to answer this question have been hampered in the past due to the use of inappropriate statistics. McKinnon (2002) notes that the picture of elliptical OPM is consistent with observations showing distributions of Stokes $V / I$ that are broad and centred near zero (Backer \& Rankin 1980; Stinebring et al. 1984a) (or equivalently, we note, that $\langle|V|\rangle$ tends to greatly exceed $|\langle V\rangle|$; Karastergiou et al. 2003). However, such a distribution could not be considered a particularly distinctive feature of OPM and could easily be produced even by a mechanism for the production of circular polarization that is independent of the linear OPM phenomenon. A much more stringent test 
is to check that the observed $V$ is, along with $Q$ and $U$, consistent with a constant orientation of $\boldsymbol{p}$. The natural complement to the position angle in this regard is the ellipticity angle, $\chi=\frac{1}{2} \tan ^{-1} V / \sqrt{Q^{2}+U^{2}}:$ together they completely specify the orientation of $\boldsymbol{p}$ via the spherical coordinate angles $2 \psi, 2 \chi$. The distributions in both parameters should be bimodal under OPM (unless one mode always dominates), unlike the distribution of $V / I$, which may be unimodal.

While a measurement of the joint probability density function, $f(\psi, \chi)$, contains sufficient information to detect the presence of elliptical OPM, this choice of parameterisation is not ideal. This is because a given solid angle element on the Poincaré sphere subtends an area in $\psi, \chi$-space that itself depends on $\chi$. Specifically,

$\mathrm{d} \boldsymbol{a}=4 \mathrm{~d} \psi \mathrm{d} \chi \cos 2 \chi$.

This means that points closer to the equator $(\chi=0)$ are given greater weight in the distribution than justified by their density on the sphere (see also McKinnon 2003b). This problem could be circumvented by using $f(\psi, \chi) / \cos 2 \chi$ instead, but the problem remains that the spatial scale with which features in the distribution are represented in Cartesian $\psi, \chi$ coordinates (for example in a plot) varies with $\chi$.

A solution to this problem is to measure $f(2 \psi, \sin 2 \chi)$, which satisfies the equal-area condition

$\mathrm{d} \boldsymbol{a}=\mathrm{d}(2 \psi) \mathrm{d}(\sin 2 \chi)$.

This choice of parameterisation is known as Lambert's cylindrical equal-area projection. In fact, any equal-area projection has the desired properties of providing true solid-angle densities and representative sizes for features. Lambert's cylindrical equal-area projection suffers from severe shape distortions at both poles $\left(Q=U=0\right.$, i.e. $\left.\chi= \pm 45^{\circ}\right)$ and we shall only make use of it in the case where one of the two dimensions is to be averaged in order to use the other along with pulse longitude in a two-dimensional display. That is, we supplement the traditional $\psi$ versus longitude density display with a $\sin 2 \chi$ versus longitude display that gives information on the distribution of ellipticity as a function of longitude.

To examine the distribution of polarization orientations at a single pulse longitude, it is desirable to have minimum (zero) distortion at both of the orientations associated with the OPMs. A cylindrical equal-area projection with its equator containing the OPM orientations would suffice, however the nature of deviations from OPM in PSR B0329+54 we describe below motivates the use of a projection where the distortion is axisymmetric about the modal orientation. The only such projection to also have the equal area property is Lambert's azimuthal equal area projection, $f(x, y)$ where

$x=\rho \cos \lambda$

$y=\rho \sin \lambda$

$\rho=2 \sin \left(\frac{\pi}{4}-\frac{\theta}{2}\right)$

and $\lambda, \theta$ are the azimuth and latitude of $\boldsymbol{p}$ in a rotated frame with $\mathbf{1}_{\boldsymbol{m}}$ as its "north" pole. This projection maps the entire sphere to a disc, with the "north" pole in the centre and the "south" pole distorted to a ring about the circumference. In order to avoid this severe distortion, we truncate the projection at $\theta<0$ and display the southern hemisphere in an adjacent projection with $-\mathbf{1}_{\boldsymbol{m}}$ as its central pole.

\subsection{Testing for orthogonality}

\subsubsection{Eigenanalysis of the covariance matrix}

Previous studies of pulsar polarization have frequently revealed position angle distributions with components that are significantly broader than that expected due to instrumental noise alone (e.g. Stinebring et al. 1984a), indicating the presence of some non-orthogonal radiation. Deriving as it does from the ratio of two noisy quantities ( $Q$ and $U$ ), the linear polarization position angle possesses complicated statistics, depending not only on the statistics of measurement noise but also on the statistics of variations in the linear polarization of the source. Although much effort has been expended modelling them (e.g. McKinnon 2003b), it is clear that as a means of detecting nonorthogonality, the PA distribution is less than ideal. The distribution of ellipticity angles suffers from the same problems, in addition to those discussed in Sect. 2.2. We suggest instead a more robust test of the consistency of polarization observations with an origin in OPM.

From the fact that OPMs contribute to the observed values of $\boldsymbol{p}$ only along a single vector, it follows that a simple and direct means of detecting non-orthogonal emission is to check the statistics of the components of $\boldsymbol{p}$ perpendicular to $\mathbf{1}_{\boldsymbol{m}}$. In order to do this, it is necessary to define a new orthogonal basis for $\boldsymbol{p}$, which has $\mathbf{1}_{\boldsymbol{m}}$ as one of the basis vectors. The question then arises of what to use for a working value of $\mathbf{1}_{\boldsymbol{m}}$, since when the orthogonality of all states is in question, the mean polarization vector may not be parallel to $\mathbf{1}_{\boldsymbol{m}}$. Under the assumption that the majority of the fluctuations in $\boldsymbol{p}$ are directed along $\mathbf{1}_{\boldsymbol{m}}$, a sensible choice for this vector is the direction of greatest variance in $\boldsymbol{p}$, which is also the least-squares estimate of the direction of fluctuations. The method of Principal Components Analysis (PCA; e.g. Jollife 1986) is suggested as a means of finding this vector. PCA is based on the fact that the set of eigenvectors of the covariance matrix of a multivariate statistic represent an orthogonal basis in which the variations in the different axes have zero covariance ${ }^{1}$. That is, given the covariance matrix

$\boldsymbol{C}=\left\langle(\boldsymbol{p}-\langle\boldsymbol{p}\rangle)(\boldsymbol{p}-\langle\boldsymbol{p}\rangle)^{T}\right\rangle$

where $\boldsymbol{p}$ is a column vector, there exists a set of 3 orthonormal vectors $\boldsymbol{e}_{\boldsymbol{i}}$ (eigenvectors) and scalars $\lambda_{i}$ (eigenvalues) such that

$C \boldsymbol{e}_{i}=\lambda_{i} \boldsymbol{e}_{i}$

\footnotetext{
1 We note that a similar technique was independently described for OPM use by McKinnon (2004) while this paper was in the final stages of preparation.
} 
It is easily shown that the covariance matrix is diagonal if the vectors are expressed using the eigenvectors as a basis. That is:

$$
\begin{aligned}
\boldsymbol{C}^{\prime} & =\left\langle[\boldsymbol{M}(\boldsymbol{p}-\langle\boldsymbol{p}\rangle)][\boldsymbol{M}(\boldsymbol{p}-\langle\boldsymbol{p}\rangle)]^{T}\right\rangle \\
& =\left(\begin{array}{ccc}
\lambda_{1} & 0 & 0 \\
0 & \lambda_{2} & 0 \\
0 & 0 & \lambda_{3}
\end{array}\right),
\end{aligned}
$$

where $\boldsymbol{M}$ is a matrix with the eigenvectors as its rows.

Clearly, the eigenvalues are equal to the variances in the components aligned with the corresponding vectors. The eigenvector with the greatest eigenvalue corresponds to our choice of $\mathbf{1}_{\boldsymbol{m}}$, while the other vectors and their associated eigenvalues present a convenient basis for detection and characterisation of non-orthogonal radiation. In theory there may be fewer than three distinct eigenvalues indicating axisymmetry (two eigenvalues) or isotropy (one eigenvalue) in the directional variance, although the presence of measurement noise makes this have zero probability in practice. However, measurement noise also introduces bias to the covariance matrix that should be corrected by subtraction of the covariance of the noise, estimated from off-pulse longitudes and including also the contribution of the pulsar to the system temperature if significant. Where the intrinsic covariance is of a similar magnitude to the uncertainty in the off-pulse covariance, this may result in negative eigenvalues, which presents a problem if $\lambda_{i}^{1 / 2}$ is to be used as a measure of the scale of intensity fluctuations, and it must be accepted that estimates will be unavailable in some longitude bins. This problem is also familiar from, for example, bias-corrected estimates of the linearly polarized intensity, $L=\left(Q^{2}+U^{2}-\sigma_{Q}^{2}-\sigma_{U}^{2}\right)^{1 / 2}$.

Having measured the variance of fluctuations in components parallel to and perpendicular to the modal orientation, it is useful to define a measure of the degree of deviation from purely linear fluctuations. After Cloude \& Pottier (1995), we define the polarization entropy as:

$H=-\sum_{i=1}^{3} P_{i} \log _{3} P_{i}, P_{i}=\frac{\lambda_{i}}{\sum_{j} \lambda_{j}}$,

where $\lambda_{i}$ is the $i$ th eigenvalue. This quantity is confined to the range $[0,1]$, where $H=0$ corresponds to fluctuations completely confined to one direction, and $H=1$ corresponds to an isotropic distribution of $\boldsymbol{p}-\langle\boldsymbol{p}\rangle$.

\subsubsection{Effects of scintillation}

The methods of the previous section work from the covariance of the observed signal, which in practice may reflect not only the intrinsic variations of the pulsar, but also the effects of the interstellar medium on the propagating signal. Of principle importance is the variable effective gain of the interstellar medium induced by two types of propagation effect, refractive and diffractive scintillation. For most pulsars, the time scale for variations due to refractive scintillation is long ( $\sim 15$ days for PSR B0329+54; Stinebring et al. 2000), so the effects can be neglected over short observations. Time scales for diffractive scintillation, on the other hand, are much shorter. In some cases the time scale may be long enough that one can simply limit the analysis to a segment of time over which the flux density is constant (e.g. McKinnon 2004), but in other cases such intervals do not last long enough to sensitively obtain representative statistics. With a diffractive scintillation time scale of $\sim 148 \mathrm{~s}$ at $328 \mathrm{MHz}$ (Cordes 1986), this is the case for the observations of PSR B0329+54 reported here.

In order to separate the (co-)variance induced by scintillation from that intrinsic to the pulsar, we employ fluctuation spectral techniques (Edwards \& Stappers 2003). We define the (longitude-resolved) polarization spectral density tensor as the element-wise product of the (discrete, vector) Fourier transform of $\boldsymbol{p}_{\boldsymbol{j}}-\left\langle\boldsymbol{p}_{\boldsymbol{j}}\right\rangle$ with its Hermitian transpose, where $j$ indexes pulse number and the longitude dependence is implicit as elsewhere in this work. That is,

$\boldsymbol{S}_{\boldsymbol{k}}=\boldsymbol{P}_{\boldsymbol{k}} \boldsymbol{P}_{\boldsymbol{k}}^{\dagger}$, where

$\boldsymbol{P}_{\boldsymbol{k}}=\frac{1}{N} \sum_{j=1}^{N} \mathrm{e}^{-2 \pi i j k / N}\left(\boldsymbol{p}_{\boldsymbol{j}}-\left\langle\boldsymbol{p}_{\boldsymbol{j}}\right\rangle\right)$,

$i=\sqrt{-1}, N$ is the number of pulses, and ${ }^{\dagger}$ denotes the Hermitian transpose. Here $k$ is a frequency index (corresponding to a frequency of $k / N$ cycles per pulse period), which is most easily interpreted if it is taken in the range $(-N / 2, N / 2]$. For convenience, in practice the observation can be broken into segments for which $\boldsymbol{S}$ is computed, and the results averaged. The tensor $\boldsymbol{S}$ contains a wealth of information about the polarization fluctuations, offering among other possibilities, a means for distinguishing different sources of variation in $\boldsymbol{p}$ via their different fluctuation frequency structure, and a chance to form the complex covariance matrix of the analytic signal and check for periodic patterns of elliptical (rather than only linear) form (Edwards 2004, in preparation). In the present context we simply make use of the fact that the sum of $\boldsymbol{S}_{\boldsymbol{k}}$ over all $k$ gives, via Parseval's theorem, the covariance matrix (Eq. (13)), while the covariance matrix of a bandpass-filtered version of the data can be computed as a sum over a suitably limited range of $k$.

The effect of scintillation on the observed signal $\boldsymbol{p}_{\boldsymbol{j}}$ is a time-varying, multiplicative gain. The observed signal can be written

$\boldsymbol{p}_{j}=\left(1+m \rho_{j}\right) \boldsymbol{p}_{j}^{i}$

where $m$ is the modulation index due to scintillation, $\rho_{j}$ is a realisation of a random process of zero mean and unit variance, and $\boldsymbol{p}^{\boldsymbol{i}}$ is the intrinsic signal. Breaking the intrinsic signal into a mean contribution plus a variable contribution, Eq. (20) can be expanded to give

$\boldsymbol{p}_{j}-\langle\boldsymbol{p}\rangle=\left(1+m \rho_{j}\right)\left(\boldsymbol{p}_{\boldsymbol{j}}^{i}-\left\langle\boldsymbol{p}^{i}\right\rangle\right)+m \rho_{j}\left\langle\boldsymbol{p}^{i}\right\rangle$.

Assuming that the instrinsic and scintillative variations are independent, the covariance matrix (Eq. (13)) becomes

$\boldsymbol{C}=\left(1+m^{2}\right) \boldsymbol{C}^{\boldsymbol{i}}+m^{2}\left\langle\boldsymbol{p}^{i}\right\rangle\left\langle\boldsymbol{p}^{i}\right\rangle^{T}$.

This shows that the uncorrected covariance matrix is biased and scaled by the presence of scintillation ${ }^{2}$. Replacing multiplications in the time domain of Eq. (21) with convolutions

\footnotetext{
2 As an aside we note that the presence of scaling implies, surprisingly, that scintillation can improve sensitivity to intrinsic variance,
} 
in the frequency domain, one finds that the vector spectrum of Eq. (19) consists of the sum of the spectrum of intrinsic variations from the mean, its convolution with the spectrum of the scintillation variations, and the convolution of the spectrum of the scintillation variations with the spectrum of $\left\langle p^{i}\right\rangle$, i.e. a scaled sinc function at zero frequency. The condition of independence implies that this sum carries over to Eq. (18) just as it did to Eq. (22). The spectral response of the scintillation variations has a characteristic width $1 / \tau$ (where $\tau$ is the scintillation timescale), corresponding to $\Delta k=N P / \tau$, where $P$ is the pulse period. In the usual case that $P / \tau \ll 1$, we see that the bias term in the observed covariance is confined to a small fraction of the spectral density tensor around zero frequency. Under the reasonable assumption that the fraction of intrinsic fluctuation energy at these frequencies is small, this region can simply be omitted in the sum over $k$ that is performed to form the covariance matrix, to eliminate the bias. The remaining scale factor $1+m^{2}$ can be obtained (given $\langle\boldsymbol{p}\rangle$ ) from the complementary sum of the spectral tensor over small $|k|$, which is over-constrained, necessitating a fit. In practice, since $m$ and (to first order) $\rho_{j}$ are independent of pulse longitude, it can be obtained easily and accurately by adding all on-pulse longitude bins in the Stokes I signal and extracting the analogous quantity from the fluctuation power spectrum of the result.

\section{Observations and discussion}

\subsection{Observations}

We used the Westerbork Synthesis Radio Telescope with the PuMa pulsar backend (Voûte et al. 2002) to observe PSR B0329+54 in a $10 \mathrm{MHz}$ band centred at $328 \mathrm{MHz}$. PuMa was configured as a digital filterbank, producing samples in all four Stokes parameters over 128 frequency channels with a sample interval of $409.6 \mu \mathrm{s}$. We found that our standard digitisation using 2 bits per sample per channel was insufficient to avoid clipping the brightest pulses, and caution that this may also have been a problem in previously published polarimetry of this and other bright pulsars. This problem was avoided by re-observation of the pulsar with 8 bits of digitisation. In offline analysis the data were corrected for instrumental polarization effects determined using the procedure described in the Appendix, followed by removal of the frequency-dependent position angle rotation caused by interstellar Faraday rotation, and samples were summed across all frequency channels after correcting for delays due to interstellar dispersion. The resultant time series was divided into segments corresponding to the apparent pulse period to give an array of 16400 pulses in 1744 pulse longitude bins, 220 of which were used in further analysis.

This pulsar is known to exhibit at least four main modes of emission, each with a different pulse profile (Bartel et al. 1982), two of which are commonly seen at low frequencies. By forming pulse profiles in sub-integrations of 100 pulses, we determined that the pulsar underwent a mode change at pulse number $\sim 12800$. The profiles formed by adding pulses $0-12500$

even when the data contain an equal balance of scintillative amplification and deamplification events. and 13000-16400 are consistent with the so-called "normal" and "abnormal" modes reported by previous authors.

\subsection{Distribution of polarization orientations}

As a first step in the characterisation of the distribution of orientations of the polarization vector $\boldsymbol{p}$, we formed frequency of occurrence histograms in position angle and $\sin 2 \chi$ (Sect. 2.2) as a function of pulse longitude. To reduce the effect of noise, only those samples with $|\boldsymbol{p}|>10 \sigma_{\mathrm{p}}$, where $\sigma_{\mathrm{p}}$ is the quadrature mean of the variance measured in Stokes $Q, U$ and $V$ in off-pulse longitude bins, were included in the histograms. The result for the normal profile mode is shown in Fig. 1.

The histograms for the abnormal mode were qualitatively very similar to those of the normal mode, upon which the remainder of the discussion will focus. However, we note as an aside that the position angle distributions appeared offset between the two profile modes. Although the presence of nonOPM means that the magnetic pole model of Radhakrishnan \& Cooke (1969) is not strictly applicable, for the purpose of quantifying the offset, after Gil \& Lyne (1995) we attempted to fit the model to position angles determined using local maxima in the histograms near to a curve made by eye to approximately fit the less distorted of the polarization modes (Fig. 1). We found that for the abnormal mode, a fit to the fiducial position angle parameter $\psi_{0}$ while using values determined from the normal mode for other parameters performed much better than a fit where $\psi_{0}$ was fixed and all others allowed to vary (rms deviation $3.13^{\circ}$ versus $3.91^{\circ}$ ), and was comparable to the best fit with all parameters allowed to vary $\left(\mathrm{rms} 3.06^{\circ}\right)$. We therefore conclude that the offset is consistent with a shift in position angle of $\sim-5.5^{\circ}$, rather than a change in the apparent viewing geometry or a longitude offset as might be induced by differential aberration and retardation.

While the position angle distribution of Fig. 1 is consistent with previously published results of lower resolution showing quasi-orthogonal modes (Gil \& Lyne 1995), the ellipticity distribution shows features of a kind never seen before in any pulsar, owing most likely to the fact that previous studies have used $V / I$ instead of ellipticity, causing OPM-related features to be washed out due to fluctuations in $|\boldsymbol{p}| / I$. Most striking is the strong right-circular polarization seen under the main central component, which has no corresponding left-circular component of equal ellipticity as would be expected if the circular polarization is due to the OPM clearly seen in the position angle distribution (Sects. 2.1 and 2.2). Also of interest is that the trailing component (longitude $\sim 64^{\circ}$ ) has a distribution that is roughly bimodal about the zero line, as expected under elliptical OPM, while the leading component (longitude $\sim 41^{\circ}$ ) appears to have a unimodal ellipticity distribution. Also apparent is that polarized emission is occasionally detected in the vicinity of pulse longitudes $33^{\circ}$ and $70^{\circ}$, corresponding to the additional emission components detected in total intensity by Gangadhara \& Gupta (2001).

Much more intriguing behaviour is made apparent when the full two-dimensional orientation distribution is considered for particular longitude ranges. In Fig. 2 we display these 

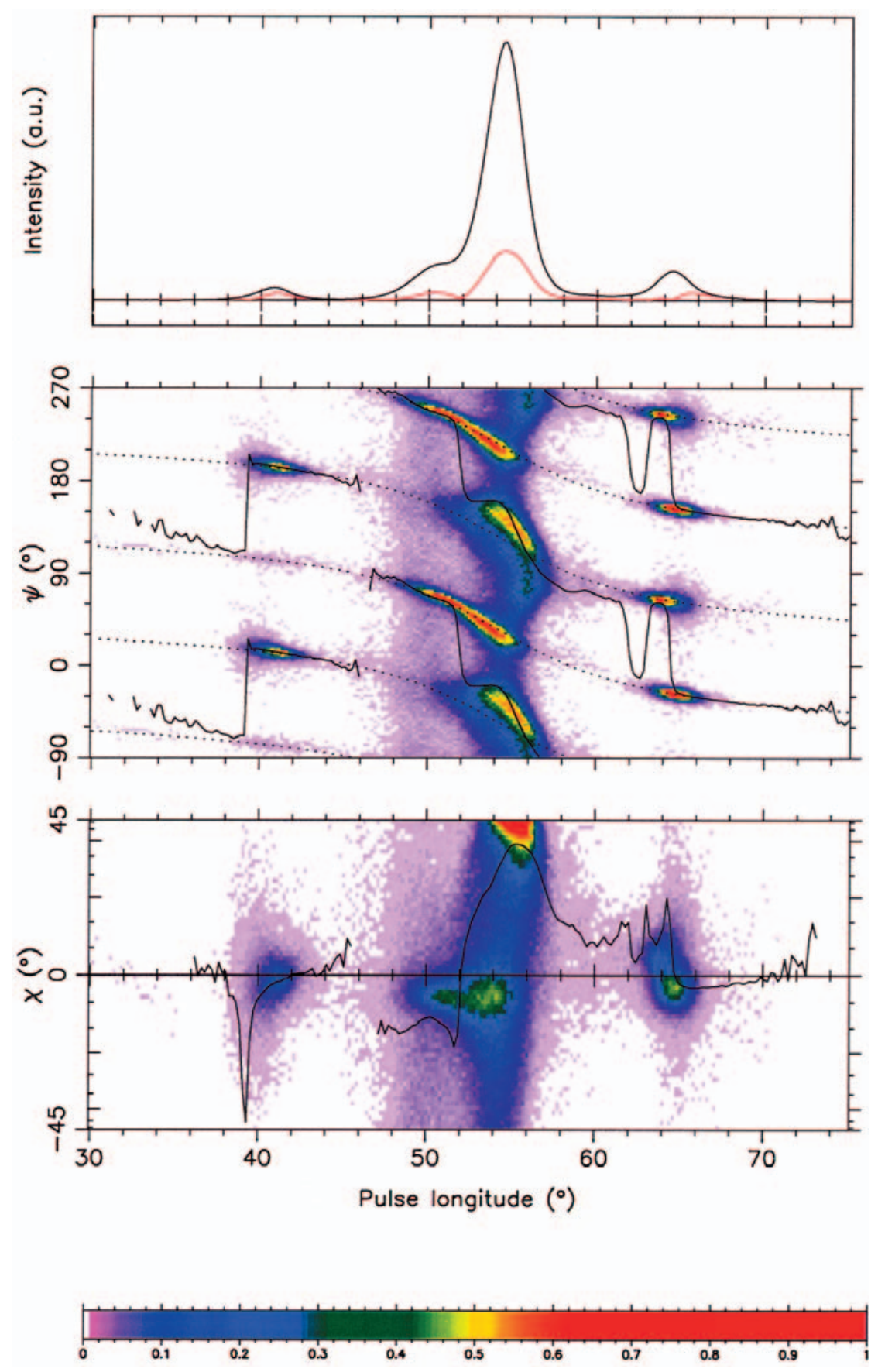

Fig. 1. Longitude-dependent polarization behaviour of PSR B0329+54 in its normal profile mode at $328 \mathrm{MHz}$. Plotted are the mean total and polarized intensity (black and red, top panel) and histograms of position angle (middle panel, plotted twice for continuity) and sin $2 \chi$ where $\chi$ is the ellipticity angle, along with the corresponding parameters of the mean polarization vector (black lines). For convenience the ordinate scale of the bottom panel is labelled non-linearly in terms of $\chi$. The histograms are normalised by the peak density and plotted in the colour scale shown at the bottom. The dashed line in the middle panel shows the prediction under the magnetic pole model (Radhakrishnan \& Cooke 1969), see text. The smooth behaviour seen is intrinsic to the pulsar, the longitude bin spacing is determined by the instrumental resolution.

distributions averaged over several longitude intervals, using the projection described in Sect. 2.2. In what follows we refer to the modes occurring in the left and right halves of each projection as modes " 1 " and " 2 " respectively. Addressing the distributions in longitude order, we see that the leading component is consistent with purely linear OPM in mode 2, while by pulse longitude $50^{\circ}$ the modes have switched in dominance and become somewhat elliptical. As the pulsar rotates, mode 2 begins to increase again in strength, and apparently has a greater spread in its orientations than mode 1 . Over the course of the central component the distribution associated with mode 2 deforms into an arc and eventually an almost complete annulus, while mode 1 remains tightly distributed around an elliptical orientation and eventually concedes dominance to mode 2. Finally, in the trailing component the modes are of comparable strength and distributed tightly around orthogonal 

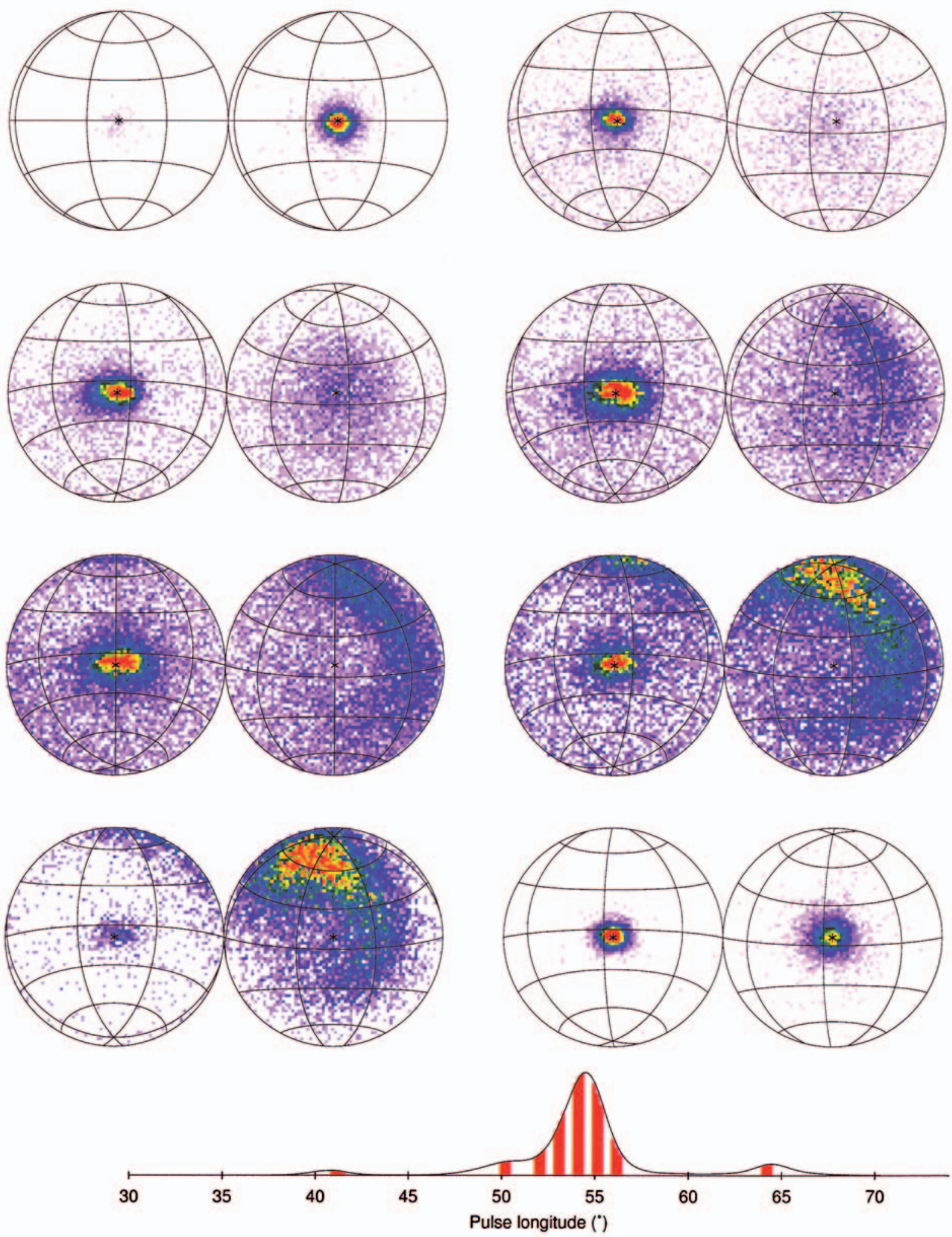

Fig. 2. Distribution of polarization orientations in eight longitude intervals, marked in the pulse profile at the bottom and shown in left-toright, top-to-bottom order. Each panel shows the distribution in Lambert's azimuthal equal-area projection with poles set near to the typical orientation of the mode showing the least scatter. The projection is interrupted at the equator and plotted in two hemispheres, to give low distortion near both poles, marked by asterisks. Lines of constant $\psi$ and $\chi$ are marked for reference; meridians (constant $\psi$ ) converge at $\chi=45^{\circ}$ (pure right-circular polarization) near the top of the projection. The colour scale is shown in Fig. 1, each density being normalised by the peak in each longitude interval. 
elliptical orientations. We discuss our interpretation of this remarkable behaviour in Sect. 3.4 but first discuss the remaining observational results.

\subsection{Covariance analysis}

The results of the previous section leave no doubt that the central component shows strong deviation from the behaviour expected under OPM. The case of the leading and trailing components is more difficult to assess due to the fact that the expected spread in orientations under instrumental noise depends in a complicated way on the distribution of $|\boldsymbol{p}|$. Instead, we used the method of eigenanalysis described in Sect. 2.3.1. In our case the dispersed pulsar signal contributes at most about one fifth of the total system temperature, justifying the use of a single, longitude-independent correction to the spectral density tensor for the off-pulse noise, under the caveat that a very small ( $\sigma \simeq 0.003$ normalized flux units) amount of measurement noise contaminates the variances for the central component. The characterstic frequency corresponding to scintillation on the diffractive time scale (148 s; Cordes 1986) is $\sim 1 / 200$ cycles per period. To ensure the response was eliminated over its full frequency extent, we excluded elements of the spectral density tensor with $|k| / N \leq 1 / 50$ when computing the covariance matrix. Using power from $|k| / N \leq 1 / 50$ in the fluctuation spectrum of the pulse energy, we measure a modulation index of 0.16 due to scintillation, in agreement with the measurements and empirical model of Cordes (1986), given our observing band. This value was used to correct the overall scale of the covariance matrix. The results of the eigenanalysis of this matrix are shown in Fig. 3.

Beginning with the polarization entropy (Eq. (17)), we see that the polarization is most disordered in the central component, as one might expect from the distributions seen in the previous sections, but still shows detectable entropy in all other pulse longitudes accessible to measurement. That the divergence from pure OPM is significant is confirmed by the fact that significant variance is detected in the second and third eigenvalues under every component. The fact that the second and third eigenvalues are nearly equal in all components except the central peak indicates that, for these pulse longitudes, the deviations from OPM show no preferred direction, and cannot be caused by position angle distortions or a random circular component alone. This is consistent with the analysis of PSR B1929+10 and PSR B2020+28 at $1404 \mathrm{MHz}$ performed by McKinnon (2004), who suggests the superposition of (isotropic) randomly polarized radiation (RPR) as the cause. On the other hand, in the central component of PSR B0329+54 all three eigenvalues are significantly different, and indeed the analysis of the directional distribution in the preceding section shows that the distribution of $\boldsymbol{p}$ cannot be ellipsoidal. This implies that the deviations are themselves associated with the production of OPM, as discussed further below.

It is also interesting to note that there is a suggestion of correspondence between transitions in the mean modal dominance (e.g. Fig. 1) and local minima in the polarization entropy (Fig. 3). This appears to be the case around pulse longitudes $\sim 42^{\circ}, \sim 52^{\circ}$, and $\sim 63^{\circ}$, however the correspondence is

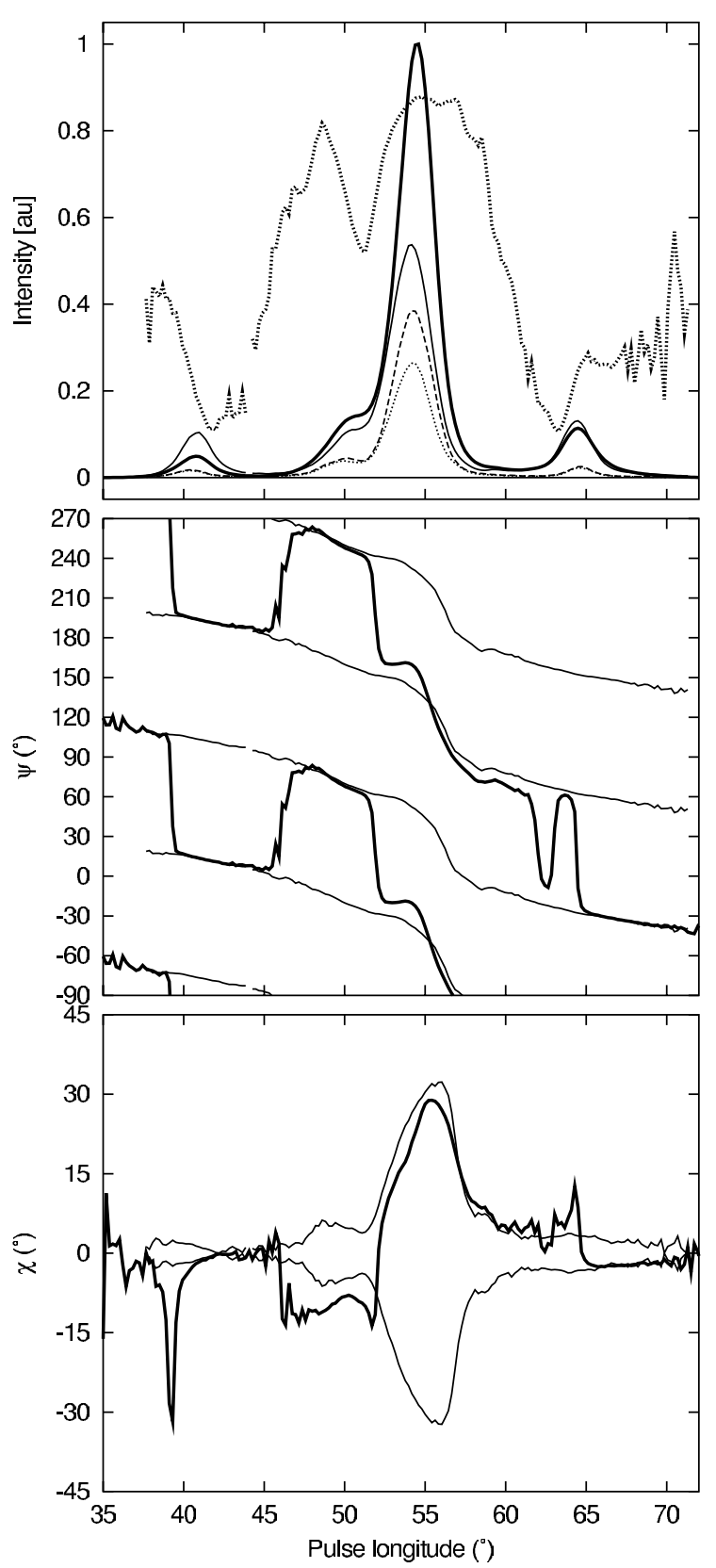

Fig. 3. Results of eigenanalysis. The top panel shows the average intensity profile (thick solid line), the square roots of the eigenvalues (solid, dashed, dotted thin lines, in descending order of value), and the polarization entropy (Eq. (17); thick dotted line). The middle panel shows the position angle of the mean polarization vector (thick line, plotted repeatedly at offsets of $180^{\circ}$ for continuity) and of the first eigenvector (thin line, plotted repeatedly at offsets of $90^{\circ}$ for continuity under the equivalence of antiparallel vectors as eigenvectors). The bottom panel shows the ellipticity angle of the mean polarization vector (thick line) along with the ellipticity angle of the first eigenvector, plotted twice with opposite signs.

not exact, particularly in the leading component. If the trend is in fact real and confirmed in other pulsars, it would require that any mechanism for the production of two OPMs predicts that under conditions leading to OPMs of similar intensity, OPMrelated fluctuations dominate more strongly over the randomly polarized fluctuations than elsewhere in the pulse profile. This 
could be the case if the modal intensities tend to be more variable or more negatively correlated (Eq. (6)), and/or the randomly polarized component is weaker or less isotropic. A detailed study of a larger sample of pulsars would be necessary to distinguish between these possibilities.

We also note that a smooth position angle curve can be constructed from the eigenvector corresponding to the largest eigenvalue, in contrast to the position angle of the average polarization vector, which shows gradual $90^{\circ}$ transitions rather than sharp flips as would be needed for reconstruction of a continuous smooth curve. Also the ellipticity angle curve of the first eigenvector avoids the problem seen at longitude $\sim 39^{\circ}$, where near complete cancelling of the linear contributions of the OPMs is not accompanied by cancelling of the circular component, giving rise to a "spike" in $\chi$ where $\langle\boldsymbol{p}\rangle /|\langle\boldsymbol{p}\rangle|$ sweeps over the left-circular pole (indicating, incidentally, nonorthogonal modes, or a consistent, superposed left-circularly polarized component). These properties will likely make eigenanalysis a useful technique for detecting polarization fluctuations driven by OPMs and determining the longitude dependence of their polarization orientations, even when the signalto-noise ratio is insufficient to detect individual pulses.

\subsection{Interpretation of strong deviations from OPM}

The results of the preceding sections show that, while at some pulse longitudes the circular polarization is typically in proportion with the linear polarization such that two clusters are produced in the orientation distribution at antipodal points on the Poincaré sphere, at no pulse longitude is the scatter in orientations consistent with instrumental noise alone. Moreover, near the peak of the average profile, one mode shows extreme divergence from the expected orientation, with a correlation between position angle and ellipticity that is complex in form. The shape is consistent with a broad, incomplete annulus centred upon the point opposite to the orientation of the other mode.

A possible origin for this behaviour lies in birefringent effects in the magnetosphere. Specifically, the annular form is suggestive of a propagation effect whereby the polarization state of incoming rays as represented on the Poincaré sphere are rotated by a time-varying angle about the axis defined by the central point of the annulus. Such an effect is expected if the observed radiation passes through a region of plasma where the natural propagation modes are different to the ray polarization, and a net phase delay occurs between the components of the electric field in each of the two modes due to their different group velocities. This effect has been termed Generalised Faraday Rotation (GFR; Kennett \& Melrose 1998) and is familiar from, but not theoretically limited to, ordinary Faraday rotation in the interstellar medium (about Stokes $V$, due to the circular modes of cold, non-relativistic magnetised plasma), and from the effect of retardation plates (rotation of $\boldsymbol{p}$ about a linear orientation defined by the optical axis of the material). In the case of the pulsar, the polarization of the plasma modes can be identified with the slightly elliptical polarization states that appear antipodal on the Poincare sphere at the centre of the annulus and at the typical orientation of states dominated by the other, well-behaved mode. The incoherent superposition of radiation in the other polarization mode, which apparently does not suffer this effect, would cause the annulus to broaden outwards, helping to explain the spread of the observed distribution.

This kind of effect was predicted for pulsar magnetospheres by Cheng \& Ruderman (1979) and given a quantitative treatment by Lyubarskii \& Petrova (1999). In their formulation the change in polarization is effected in the vicinity of the polarization limiting region (PLR; Sect. 1), where the plasma density is insufficient to cause total decoherence of the modes, yet densities are still high enough to cause significant phase delays between the modes. Radiation enters this region as an incoherent mixture of the local plasma modes, but due to changes in the modal orientation along the ray path caused by the rotation of the magnetosphere, each of the incoming rays acquires components in both of the propagation modes, which propagate at different speeds and alter the polarization of the ray accordingly. This picture deviates from our observations in several ways. Firstly, Lyubarskii \& Petrova (1999) assume linearly polarized propagation modes, whereas the observations indicate elliptical modes, implying plasma with a net charge density rather than a pure pair plasma. Secondly, the predicted effect is not as simple as rotation about a given axis, for the modal polarization orientation, and thus the axis of rotation, varies along the ray path. The presence of an annular shape, as expected from a near-constant polarization of the propagation modes, may therefore place some constraints on the size of the region of the magnetosphere contributing significant, variable amounts of GFR. Finally, the effect should only be capable of inducing one sense of circular polarization (Radhakrishnan \& Rankin 1990; Lyubarskii \& Petrova 1999), and should affect both rays equally apart from a reversal in the sense of circular polarization (Petrova 2001).

An alternative cause of the misalignment of the polarization of the propagation modes and the incoming rays, is refraction. Petrova \& Lyubarskii (2000) show that, while the extraodinary mode propagates under a vacuum dispersion law, the ordinary mode can suffer from significant refraction, which, under a non-axisymmetric plasma distribution, can cause it to move out of the plane of the magnetic field line from which it originated (and obtained its initial polarization). The calculations of Petrova \& Lyubarskii (2000) show that the subsequent alteration of the polarization state at the PLR can produce either sense of circular polarization, as seen in our observations. Moreover, since the extraordinary mode is immune to refraction, it should not suffer the same PLR effects, consistent with the tight, centrally peaked distributions of orientations observed here in mode 1 . Should one of the modes be produced by conversion from the other, as in Petrova (2001), this would imply that refraction occurs above the conversion region. An alternative means of producing mode-dependent PLR effects is the invocation of [anti-]correlation between the efficiency of conversion and the physical conditions in the PLR (Petrova 2001), however for this to be the case the correlation must be very strong, given the complete absence of an annulus in the distribution of states apparently dominated by mode 2 . That refraction-driven PLR effects only occur close to the magnetic 
axis (Petrova \& Lyubarskii 2000) is another prediction borne out by the observation that only the central profile component shows the annular distribution. Many pulsars show strong mean circular polarization in central, so-called "core" components (Rankin 1983), which tends to show a central sense reversal (Radhakrishnan \& Rankin 1990, although see also Han et al. 1998) taken by Petrova \& Lyubarskii (2000) as support for their model of the refraction-driven PLR effect. The probable direct detection of this effect in PSR B0329+54 opens the possibility of good tests of the model through applications of the techniques used here on a larger sample of pulsars with and without "core" components, and examination of the frequency dependence.

Detailed modelling of this effect is beyond the scope of this work, however to prove the basic assertion that GFR can produce the spread of orientations observed, we have performed some basic numerical simulations. We simulated the observed polarization vector as the sum of three components, the ordinary ray, the extraordinary ray and an RPR component:

$p=p_{0}+p_{\mathrm{e}}+p_{\mathrm{r}}$

We assume that certain parameters are independently distributed according to chosen "reasonable" distributions. These are merely intended to provide variation in order to produce the qualitative features of the model, and are not expected to necessarily closely resemble the distributions under a complete physical model. The ordinary mode was presumed to have an initially linear polarization orientation with a position angle drawn from a von Mises distribution:

$f(2 \psi)=\frac{\mathrm{e}^{\kappa \cos \left(2 \psi-\mu_{\mathrm{v}}\right)}}{2 \pi I_{0}(\kappa)}$

(where $I_{0}$ is a modified Bessel function of the first kind) with $\mu_{\mathrm{v}}=50^{\circ}, \kappa=20$, while its (polarized) intensity was drawn from an exponential distribution:

$f(I)= \begin{cases}\frac{1}{\mu} \mathrm{e}^{-I / \mu_{\mathrm{e}}} & I \geq 0 \\ 0 & I<0,\end{cases}$

with $\mu_{\mathrm{e}}=1$. To model the effect of GFR, the initial state for the ordinary mode was rotated by an angle $\theta$ about an axis $\mathbf{1}_{m}$ oriented with $\psi=90^{\circ}, \chi=4^{\circ}$, where $\theta$ was also drawn from a von Mises distribution $\left(\mu_{\mathrm{v}}=90^{\circ}, \kappa=1\right)$, to give the computed $\boldsymbol{p}_{\mathbf{0}}$. The extraordinary mode $\left(\boldsymbol{p}_{\mathbf{e}}\right)$ was presumed to be always oriented with $\psi=0^{\circ}, \chi=-4^{\circ}$, with a (polarized) intensity drawn from an exponential distribution of mean $\mu_{\mathrm{e}}=0.2$. The contribution of RPR $\left(\boldsymbol{p}_{\mathbf{r}}\right)$ was calculated by drawing its components $Q, U, V$ from independent Gaussian distributions:

$f(x)=\frac{1}{\sigma \sqrt{2 \pi}} \mathrm{e}^{\frac{\left(x-\mu_{g}\right)^{2}}{2 \sigma^{2}}}$,

with $\mu_{\mathrm{g}}=0$ and $\sigma=0.05$. This model was realised $10^{6}$ times to produce the distribution of orientations depicted in Fig. 4. Although clearly the chosen distributions are not perfect, the resemblance to the observed distributions is striking, confirming the possibility that GFR is in effect.

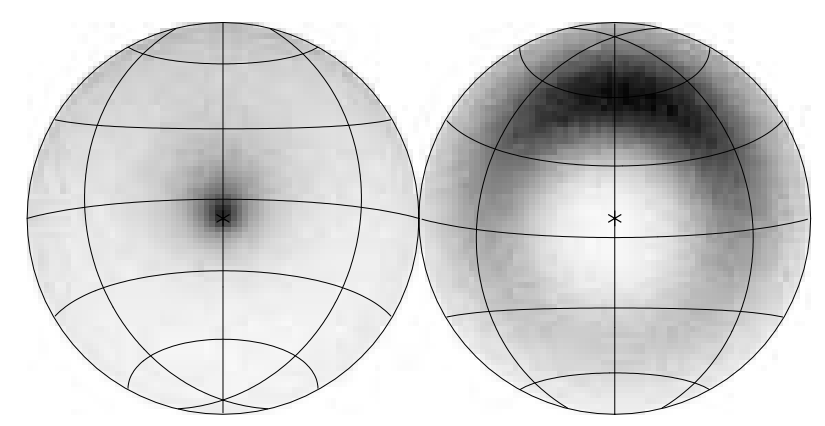

Fig. 4. Distribution of polarization orientations deriving from a simulation involving GFR (see text). The projection used is as in Fig. 2, plotted in a linear grey density scale.

\section{Conclusions}

We have performed a detailed analysis of the polarization of individual pulses from PSR B0329+54 at $328 \mathrm{MHz}$. The use of statistics involving the ellipticity angle instead of Stokes $V$ or $V / I$ enabled us to reveal for the first time the rich structure of the circular polarization distribution, which is correlated with the position angle distribution.

Displaying the distribution of polarization orientations in an equal-area projection, a remarkable structure was uncovered for pulse longitudes near the centre of the average pulse. The annulus-like form of the distribution in one mode is, in our view, indicative of Generalised Faraday Rotation (GFR) in the pulsar magnetosphere, while the fact that it apparently only affects one polarization mode, and is capable of producing either sense of circular polarization, is taken as an indication that refraction of the ordinary mode is taking place. The apparent ellipticity of the polarization state upon which the annulus centres and orthogonal to the typical polarization of states dominated by the other mode are taken to indicate that the GFR occurs in a region with elliptically polarized plasma propagation modes, indicating a net charge density in the plasma.

Through the analysis of the covariance of the Stokes parameters, by means of eigenvector decomposition, we have shown quantitatively for the first time that the circular polarization is consistent with an origin in elliptically polarized orthogonal polarization modes. Moreover, we find that a significant apparently randomly polarized component dilutes the purity of OPM-driven variations in polarization state, as also found recently by McKinnon (2004) for PSR B1929+10 and PSR B2020+28 at $1404 \mathrm{MHz}$.

In this work we have shown how to detect and characterise deviations from OPM in more powerful ways than previously available. Future application of these techniques to other pulsars over a broad frequency range should allow renewed progress in the resolution of decades-long debate about the origin of pulsar polarization.

Acknowledgements. The authors thank W. van Straten for fruitful discussions. For their useful comments on the manuscript we thank S. Petrova, and the referee who raised issues that led to important improvements. R.T.E. is supported by a NOVA fellowship. 
The Westerbork Synthesis Radio Telescope is administered by ASTRON with support from the Netherlands Organisation for Scientific Research (NWO).

\section{References}

Allen, M., \& Melrose, D. 1982, Proc. Astr. Soc. Aust., 4, 365

Backer, D. C., \& Rankin, J. M. 1980, ApJS, 42, 143

Backer, D. C., Rankin, J. M., \& Campbell, D. B. 1976, Nature, 263, 202

Bartel, N., Morris, D., Sieber, W., \& Hankins, T. H. 1982, ApJ, 258, 776

Britton, M. C. 2000, ApJ, 532, 1240

Cheng, A. F., \& Ruderman, M. 1979, ApJ, 229, 348

Cloude, S. R., \& Pottier, E. 1995, Opt. Eng., 34, 1599

Cordes, J. M. 1986, ApJ, 311, 183

Cordes, J. M., Rankin, J. M., \& Backer, D. C. 1978, ApJ, 223, 961

Edwards, R. T., \& Stappers, B. W. 2003, A\&A, 407, 273

Gangadhara, R. T., \& Gupta, Y. 2001, ApJ, 555, 31

Gil, J. A., \& Lyne, A. G. 1995, MNRAS, 276, L55

Hamaker, J. P. 2000, A\&AS, 143, 515

Hamaker, J. P., Bregman, J. D., \& Sault, R. J. 1996, A\&AS, 117, 137

Han, J. L., Manchester, R. N., Xu, R. X., \& Qiao, G. J. 1998, MNRAS, 300,373

Johnston, S. 2002, Publ. Astr. Soc. Aust., 19, 277

Jollife, T. 1986, Principal Component Analysis (New York: Springer-Verlag)

Jones, C. R. 1941, J. Opt. Soc. Am., 31, 488

Karastergiou, A., Johnston, S., Mitra, D., van Leeuwen, A. G. J., \& Edwards, R. T. 2003, MNRAS, 344, L69
Kennett, M., \& Melrose, D. 1998, Proc. Astr. Soc. Aust., 15, 211

Lyubarskii, Y. E., \& Petrova, S. A. 1999, Astrophys. Space Sci., 262, 379

Manchester, R. N., Taylor, J. H., \& Huguenin, G. R. 1975, ApJ, 196, 83

McKinnon, M. M. 2002, ApJ, 568, 302

McKinnon, M. M. 2003a, ApJ, 590, 1026

McKinnon, M. M. 2003b, ApJS, 148, 519

McKinnon, M. M. 2004, ApJ,in press [arXiv: astro-ph/0401598]

McKinnon, M. M., \& Stinebring, D. R. 2000, ApJ, 529, 435

Petrova, S. A. 2001, A\&A, 378, 883

Petrova, S. A. 2003, A\&A, 408, 1057

Petrova, S. A., \& Lyubarskii, Y. E. 2000, A\&A, 355, 1168

Radhakrishnan, V., \& Cooke, D. J. 1969, Astrophys. Lett., 3, 225

Radhakrishnan, V., \& Rankin, J. M. 1990, ApJ, 352, 258

Rankin, J. M. 1983, ApJ, 274, 333

Sault, R. J., Hamaker, J. P., \& Bregman, J. D. 1996, A\&AS, 117, 149

Stinebring, D. R., Cordes, J. M., Rankin, J. M., Weisberg, J. M., \& Boriakoff, V. 1984a, ApJS, 55, 247

Stinebring, D. R., Cordes, J. M., Weisberg, J. M., Rankin, J. M., \& Boriakoff, V. 1984b, ApJS, 55, 279

Stinebring, D. R., Smirnova, T. V., Hankins, T. H., et al. 2000, ApJ, 539,300

van Straten, W. 2002, ApJ, 568, 436

van Straten, W. 2004, ApJS, 152, 129

Voûte, J. L. L., Kouwenhoven, M. L. A., van Haren, P. C., et al. 2002, A\&A, 385, 733

von Hoensbroech, A., Lesch, H., \& Kunzl, T. 1998, A\&A, 336, 209

Weiler, K. W., \& Wilson, A. S. 1977, A\&A, 58, 17 
R. T. Edwards and B. W. Stappers: Ellipticity and deviations from OPM in PSR B0329+54, Online Material p 1

\section{Online Material}




\section{Appendix A: Polarization calibration}

The Westerbork Synthesis Radio Telescope (WSRT) is an array of 14 dishes, each of two nominally linear dipoles, which is configured as a tied array during pulsar or VLBI observations. Assuming that the response of each telescope to the incident electric field is linear, the observed voltages can be described using a Jones matrix (Jones 1941) for each dish, $i$ :

$\boldsymbol{v}_{i}=\boldsymbol{J}_{i} \boldsymbol{e}$.

The procedure for forming a tied array is to add the voltages of all telescopes after correcting them for relative phase (due to the source direction):

$\boldsymbol{v}_{\text {tot }}=\sum_{i=1}^{N} \mathrm{e}^{-i \theta_{i}} \boldsymbol{J}_{i} \boldsymbol{e}$.

where $N$ is the number of telescopes. It is therefore trivially true that the tied array signal can be calibrated by determining an overall system Jones matrix, i.e.:

$$
\begin{aligned}
& \boldsymbol{v}_{\mathrm{tot}}=\boldsymbol{J}_{\mathrm{tot}} \boldsymbol{e}, \\
& \boldsymbol{J}_{\mathrm{tot}}=\sum_{i=1}^{N} \mathrm{e}^{-i \theta_{i}} \boldsymbol{J}_{i} .
\end{aligned}
$$

The problem of tied array calibration is thus no different to the calibration of single-dish observations, for which several recent treatments are available that avoid potentially dangerous simplifying assumptions of earlier works (Hamaker et al. 1996; Sault et al. 1996; Hamaker 2000; Britton 2000; van Straten 2002; Johnston 2002; van Straten 2004). These approaches are based on the Jones matrix formalism, which has seven degrees of freedom in the instrumental model of each frequency channel (the eighth, absolute phase, is lost in the computation of the self coherency matrix for single dish or tied array observations). Hamaker (2000) divides these into a "polrotation", which causes a pure rotation of $\boldsymbol{p}$ about an arbitrary axis by an arbitrary angle (3 degrees of freedom), a "poldistortion", which exchanges an arbitrary amount of power between Stokes I and and an arbitrarily oriented component of $\boldsymbol{p}$ (3 degrees of freedom), and an overall system gain (one degree of freedom). A similar decomposition was made independently by Britton (2000), who notes that Jones matrix transformations are isomorphic with the Lorentz group, with the type of transformation called a "poldistortion" by Hamaker (2000) corresponding to a Lorentz boost. Apart from providing a convenient geometric interpretation of the kinds of transformations effected by linear system components, as Hamaker (2000) notes this decomposition suggests a calibration strategy that begins with the observation of a presumed unpolarized source, immediately giving the gain and poldistortion. The polrotation can then be obtained by observations of a further two sources about which some assumptions can safely be made (for example, that they are linearly polarized and/or have a given position angle).

Unfortunately efforts to calibrate the WSRT in tied array mode are severely hampered by the inclusion of non-linear system components. Specifically, the voltage signals from each telescope are sampled using thresholds that are dynamically determined using a control system that attempts to maintain the variances of the sampled signals at a constant level, based on averaging with some time constant of the order $1 \mathrm{~ms}$. These are known as the automatic gain controllers (AGCs). Although these components might at first appear disastrous for the detection of non-stationary signals, the fact that they normalise the individual telescope powers while the source signal adds coherently means that the distortion is reduced for $N \gg 1^{3}$. However, the fact remains that the AGCs introduce an unknown, timevarying gain to each of the real and imaginary parts of each polarization channel of each telescope, making precise calibration impossible for tied array observations, and we strongly suggest that future telescope arrays provide tied-array systems free of AGCs. Since the system is not precisely calibratable, we limit the scope of our calibration to the grossest of instrumental effects, differential gain and phase between the summed $X$ and $Y$ polarization channels. Remaining effects are expected in any case to be small, due to the high accuracy of dipole setting at WSRT (Weiler \& Wilson 1977), and the tendency for random error terms to cancel in the sum of Eq. (A.4). By comparison of our observations with published polarimetric profiles available via the EPN database ${ }^{4}$, we estimate that our results are accurate to within a few percent, certainly sufficient for examination of the basic polarization properties of the source.

The procedure we use for determining the differential phase and gain is based on the differential Faraday rotation across the observing band. This provides a variety of input polarization states incident on the telescope, with a known relationship between them, allowing one to fit simultaneously for the source polarization and the instrumental response. In this regard the technique is similar to previous strategies employing the parallactic angle rotation during long observations of a source (Stinebring et al. 1984a), which cannot be used at the WSRT because its equatorial mounts cause the dipoles to track the parallactic rotation (by the same token, this eliminates a potential source of time-variability in incompletely calibrated measurements). Just as the parallactic technique is limited to the assumption that neither the source polarization nor the telescope response changes with time, our method assumes frequency independence except for an overall factor incorporating the gain and intrinsic intensity spectrum of the source. The technique is also limited by the commutativity of certain transformations with the known transformation effected by Faraday or parallactic rotation, about Stokes $V$. That is, for any solution of the system Mueller matrix (which is constructed from the Jones matrix, see e.g. Hamaker et al. 1996) satisfying

$\boldsymbol{S}_{i}^{\prime}=\boldsymbol{M} \boldsymbol{R}_{\boldsymbol{V}}\left(2 \theta_{i}\right) \boldsymbol{S}$,

where $\boldsymbol{S}$ is the intrinsic Stokes 4 -vector, $\boldsymbol{S}_{i}^{\prime}$ is a measured (uncalibrated) Stokes 4-vector observed under a known Faraday or parallactic rotation angle $\theta_{i}$ and $\boldsymbol{R}_{\boldsymbol{V}}\left(2 \theta_{i}\right)$ is a Mueller matrix effecting a rotation of angle $2 \theta_{i}$ about Stokes $V$, there exists an infinite number of other solutions

$\boldsymbol{S}_{i}^{\prime}=\boldsymbol{M E R}_{\boldsymbol{V}}\left(2 \theta_{i}\right) \boldsymbol{E}^{-1} \boldsymbol{S}$

3 See the PuMa manual,

http://www . astron.nl/wsrt/WSRTobs/PuManual.pdf

4 http://www .mpifr-bonn.mpg.de/div/pulsar/data/ 
R. T. Edwards and B. W. Stappers: Ellipticity and deviations from OPM in PSR B0329+54, Online Material p 3

where $\boldsymbol{E}$ is any matrix that commutes with $\boldsymbol{R}_{\boldsymbol{V}}\left(2 \theta_{i}\right)$. Without additional constraints, this approach is therefore only able to determine the system response to within an arbitrary rotation ("polrotation") and boost ("poldistortion") in Stokes $V$. However, as stated above we have chosen to use a reduced description of the system response, parameterised only by frequency-dependent gain, and X-Y differential gain and phase. For assumed perfect linear dipoles, differential phase and gain errors correspond to rotations and boosts about Stokes $Q$ (e.g. Britton 2000), which do not commute with $\boldsymbol{R}_{\boldsymbol{V}}$, obviating the need for additional constraints. Our model is thus:

$\boldsymbol{S}^{\prime}(v)=g(v) \boldsymbol{R}_{\boldsymbol{Q}}\left(2 \mathrm{RM}(c / v)^{2}+\theta\right) \boldsymbol{B}_{\boldsymbol{Q}}(\beta) \boldsymbol{S}+$ noise,

where $g(v)$ models the frequency dependent gain and source spectrum, RM is the rotation measure of the propagation path between the source and Earth, $\theta$ is the phase offset between $\mathrm{X}$ and Y channels, and $\beta=\ln g_{x} / g_{y}$ where $g_{x}$ and $g_{y}$ are the (voltage) gains of the $X$ and $Y$ channels.

We define the best-fit system response as the one that minimises the global $\chi^{2}$ :

$\chi^{2}=\sum_{i}\left(\frac{\boldsymbol{D}_{i I}^{2}}{\sigma_{I, i}^{2}}+\frac{\boldsymbol{D}_{i Q}^{2}}{\sigma_{Q, i}^{2}}+\frac{\boldsymbol{D}_{i U}^{2}}{\sigma_{U, i}^{2}}+\frac{\boldsymbol{D}_{i V}^{2}}{\sigma_{V, i}^{2}}\right)$,

where $i$ indexes all measured Stokes vectors (i.e. all frequency channel/pulse longitude pairs), $\sigma_{\{I Q U V\}}^{2}$ are the variances of each of the Stokes parameters in off-pulse regions (potentially also dependent on $i$ ), and

$\boldsymbol{D}_{i}=\boldsymbol{S}_{i}^{\prime}-\boldsymbol{M}_{i} \boldsymbol{R}_{V}\left(2 \theta_{i}\right) \boldsymbol{S}$,

where in our case, $\boldsymbol{M}_{i}=g\left(v_{i}\right) \boldsymbol{R}_{\boldsymbol{Q}}\left(2 \mathrm{RM}\left(c / v_{i}\right)^{2}+\theta\right) \boldsymbol{B}_{\boldsymbol{Q}}(\beta)$. In the general case where a full system calibration is achievable $\boldsymbol{M}$ should be parameterised as a function of frequency by the seven real and imaginary components of the elements of the Jones matrix (the imaginary part of one of the diagonals can be set to zero since absolute phase is irrelevant). Johnston (2002) describes a similar scheme to that described above, employing a Jones matrix formalism and parallactic angle rotation, from which we differ in several respects. Firstly rather than minimising $\chi^{2}$, which corresponds to the maximum likelihood estimator, Johnston (2002) minimises the Manhattan distance, which is likely to have poorer estimation accuracy than maximum likelihood. Secondly, Johnston (2002) discusses the difficulties of multi-step calibration schemes but does not propose an alternative. Including all measurements of all sources in the global $\chi^{2}$, including any injected noise sources and continuum calibrators, and minimising it in one step is in our view the best way to avoid these difficulties. Finally, we caution against

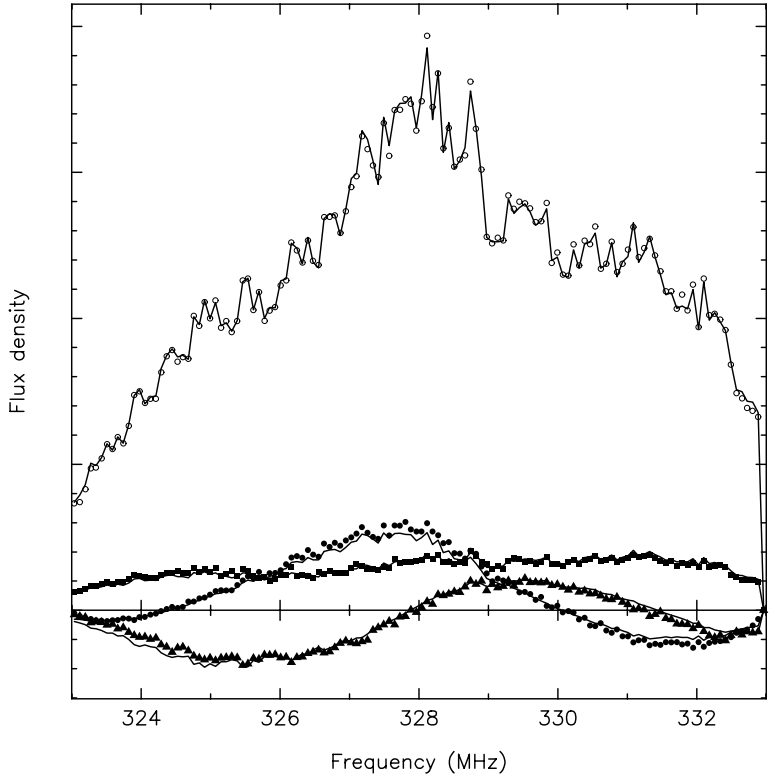

Fig. A.1. Observed values of Stokes $I$ (open circles), $Q$ (triangles), $U$ (squares) and $V$ (filled circles) in the peak longitude bin of PSR B0329+54, as a function of frequency. Predictions of the bestfitting model (incorporating all longitude bins) are shown with lines.

neglecting second-order terms in the expansion of the Mueller matrix as it is unnecessary and introduces the possibility for error if and when the technique is applied to telescopes with non-negligible cross terms (e.g. LOFAR/SKA). We note that the issues described above have received independent treatment in the recent work of van Straten (2004), who also provides a method for minimization of Eq. (A.8) that avoids explicit expansion of the Mueller matrix and its partial derivatives.

We performed the procedure described above using a frequency-resolved polarimetric profile from the first 12500 pulses of the observation of PSR B0329+54 described in the text. That $\theta \simeq \pm 90^{\circ}$ is clearly visible in the frequency dependence of the Stokes parameters (Fig. A.1), where the Faraday modulation appears mainly in $Q$ and $V$, instead of $Q$ and $U$ as expected if $\theta=0$. In fact it was this feature, seen in this and other WSRT observations in the $328 \mathrm{MHz}$ band that led us to the calibration scheme described here. The results of the fit support the assertion that $\theta \neq 0$, yielding $\theta=-75.24^{\circ}$ and $\beta=-0.0278$, implying $g_{x} / g_{y}=0.973$ (the model predictions using these values and the other parameters are plotted in Fig. A.1). These values were used to correct the recorded Stokes vectors before further analysis described in the body of this paper. 